\title{
GOLUBEV SERIES FOR SOLUTIONS OF ELLIPTIC EQUATIONS
}

\author{
CH. DORSCHFELDT AND N. N. TARKHANOV
}

\begin{abstract}
Let $P$ be an elliptic system with real analytic coefficients on an open set $X \subset \mathbb{R}^{n}$, and let $\Phi$ be a fundamental solution of $P$. Given a locally connected closed set $\sigma \subset X$, we fix some massive measure $m$ on $\sigma$. Here, a non-negative measure $m$ is called massive, if the conditions $s \subset \sigma$ and $m(s)=0$ imply that $\overline{\sigma \backslash s}=\sigma$. We prove that, if $f$ is a solution of the equation $P f=0$ in $X \backslash \sigma$, then for each relatively compact open subset $U$ of $X$ and every $1<p<\infty$ there exist a solution $f_{e}$ of the equation in $U$ and a sequence $f_{\alpha}\left(\alpha \in \mathbb{N}_{0}^{n}\right)$ in $L^{p}(\sigma \cap U, m)$ satisfying $\left\|\alpha ! f_{\alpha}\right\|_{L^{p}(\sigma \cap U, m)}^{1 /|\alpha|} \rightarrow 0$ such that $f(x)=f_{e}(x)+\sum_{\alpha} \int_{\sigma \cap U} D_{y}^{\alpha} \Phi(x, y) f_{\alpha}(y) d m(y)$ for $x \in U \backslash \sigma$. This complements an earlier result of the second author on representation of solutions outside a compact subset of $X$.
\end{abstract}

\section{IntRodUCTION AND STATEMENT OF THE MAIN RESUltS}

1.1. Let $P$ be a $(k \times k)$-matrix of scalar partial differential operators with real analytic coefficients on an open set $X \subset \mathbb{R}^{n}$. Suppose further that $P$ has a fundamental solution $\Phi$ which is real analytic outside the diagonal $\Delta$ of $X \times X$. By definition, $\Phi(x, y)$ is a $(k \times k)$-matrix of distributions on $X \times X$ satisfying

$$
\left\{\begin{array}{l}
P\left(x, D_{x}\right) \Phi(x, y)=\delta(x-y) I_{k}, \\
P^{\prime}\left(y, D_{y}\right) \Phi(x, y)=\delta(x-y) I_{k}
\end{array}\right.
$$

where $P^{\prime}$ is the transposed operator to $P$, and $I_{k}$ is the identity $(k \times k)$-matrix.

Recall that, according to a theorem of Malgrange, every elliptic differential operator with real analytic coefficients on $X$ has a fundamental solution with the desired properties.

1.2. If $U$ is an open subset of $X$, then denote by $S_{P}(U)$ the vector space of all weak solutions of the system $P f=0$ on $U$. Note that because of the analytic hypoellipticity of $P$, the solutions in $S_{P}(U)$ are actually real analytic functions in $U$. For a closed subset $\sigma$ of $X$, solutions $f \in S_{P}(X \backslash \sigma)$ will be said to have singularities on $\sigma$.

In this article, we are interested in representations of solutions of the equation $P f=0$ in $X$ having singularities on a closed subset $\sigma$ of $X$. Before stating our principal result, we must first introduce one technical definition.

A (nonnegative) measure $m$ on $\sigma$ is said to be massive, if the two conditions $s \subset \sigma$ and $m(s)=0$ imply that $\overline{\sigma \backslash s}=\sigma$. In other words, every subset of $\sigma$ of

Received by the editors February 15, 1995 and, in revised form, November 20, 1996.

1991 Mathematics Subject Classification. Primary 35A20, 35C10.

Key words and phrases. Solutions with singularities, real analytic coefficients, elliptic systems, Golubev series.

This research was supported in part by the Alexander von Humboldt Foundation. 
$m$-measure zero has empty interior. As the following example shows, a massive measure exists on every closed set $\sigma$.

Example 1.1. Let $\left\{y_{j}\right\}_{j \in \mathbb{N}}$ be a sequence of points of $K$, which is dense as a set in $\sigma$. Choose a sequence of positive numbers $\left\{\mu_{j}\right\}$ such that $\sum \mu_{j}<\infty$. For a set $s \subset \sigma$, we define $m(s)=\sum_{y_{\nu} \in s} \mu_{\nu}$. Then $m$ is a massive measure on $\sigma$.

Let us fix some massive measure $m$ on $\sigma$. Our main result is the following:

Theorem 1.1. Assume that $K$ is a locally connected compact subset of $\sigma$, and $1<p<\infty$. Then for each solution $f \in S_{P}(X \backslash \sigma)$ there exist both a solution $f_{e} \in S_{P}((X \backslash \sigma) \cup \stackrel{\circ}{K})$ and a sequence $\left\{c_{\alpha}\right\}_{\alpha \in \mathbb{N}_{0}^{n}} \subseteq\left[L^{p}(K, m)\right]^{k}$ such that

$$
f(x)=f_{e}(x)+\sum_{\alpha \in \mathbb{N}_{0}^{n}} \int_{K} D_{y}^{\alpha} \Phi(x, y) c_{\alpha}(y) d m(y)
$$

holds for all $x \in X \backslash \sigma$. Furthermore, $\left\|\alpha ! c_{\alpha}\right\|_{L^{p}(K, m)}^{1 / \alpha \mid} \rightarrow 0$ when $|\alpha| \rightarrow \infty$.

We emphasize that $\stackrel{\circ}{K}$ is the interior of $K$ on $\sigma$, i.e., in the induced topology of $\sigma$.

1.3. For holomorphic functions of one variable (i.e. for the operator $P=\partial / \partial \bar{z}$ in $\mathbb{C}^{1}$ ), for compact $\sigma$ and $p=2$, Theorem 1.1 is due to Havin [7]. Havin called the corresponding representation of the form (1) Golubev-series, since it was V.V. Golubev who posed the question whether such a formula held for every function analytic in $\hat{\mathbb{C}} \backslash K$ when $K$ is a rectifiable simple arc and $m$ the Lebesgue measure on $K$. For further details on the history of the problem cf. Havin [8]. More generally, we call representations of the form (1) Golubev-series expansions for solutions with singularities.

Baernstein [1] proved an analogous representation formula for functions holomorphic off the real axis. Using complex analysis and Hilbert space methods, the second author [15] showed Theorem 1.1 for the case of compact $\sigma$ and $p=2$ (see also [16]). Simonova [13] obtained an analogous representation theorem for functions harmonic off a hyperplane. Fischer and Tarkhanov [4] constructed a Golubev-series expansion for solutions of homogeneous elliptic systems with constant coefficients in $\mathbb{R}^{n}$, having singularities on a plane of a smaller dimension. They also derived Theorem 1.1 for the case of smooth $\sigma$ and asked whether a result as formulated in Theorem 1.1 held for arbitrary locally connected sets $\sigma$.

The local connectedness of the compact set $K$ we look at is a very delicate point in the literature. In fact it is related to the problem of extension of analytic functions on a neighborhood of $K$. (See Havin [8], Varfolomeev [17] and Rogers/Zame [12].)

In this paper, we prove the result by generalizing the ideas used in [15] in an appropriate way. Since the article [15] is in Russian and does not seem to be easily available, we have decided to present the paper in a self-contained way and do not use [15] as a reference.

1.4. The converse statement to Theorem 1.1 is quite easy to prove.

Lemma 1.1. Let $K$ be a relatively compact subset of $\sigma$, and $1 \leq p<\infty$. For every sequence $\left\{c_{\alpha}\right\}_{\alpha \in \mathbb{N}_{0}^{n}} \subset\left[L^{p}(K, m)\right]^{k}$, satisfying $\left\|\alpha ! c_{\alpha}\right\|_{L^{p}(K, m)}^{1 /|\alpha|} \rightarrow 0$ when $|\alpha| \rightarrow \infty$, the series $\sum_{\alpha} \int_{K} D_{y}^{\alpha} \Phi(x, y) c_{\alpha}(y) d m(y)$ converges for $x \in X \backslash K$ and defines an element in $S_{P}(X \backslash K)$. 
Proof. First note that for $x \in X \backslash K$ we have

$$
P(x, D) \int_{K} D_{y}^{\alpha} \Phi(x, y) c_{\alpha}(y) d m=\int_{K} D_{y}^{\alpha}\{P(x, D) \Phi(x, y)\} c_{\alpha}(y) d m=0 .
$$

Thus the proof will be complete if we show that the series we look at converges uniformly on compact subsets of $X \backslash K$. It is well-known that a $C^{\infty}$ function $g$ on an open set $U \subset \mathbb{R}^{n}$ is real analytic if and only if for every compact set $K \subset U$ there are constants $a=a(g, K)$ and $c=c(g, K)$ such that

$$
\sup _{y \in K}\left|D^{\alpha} g(y)\right| \leq c \cdot a^{|\alpha|}|\alpha| \text { ! for all } \alpha \in \mathbb{N}_{0}^{n} .
$$

Now fix a compact set $\tilde{K} \subset \subset X \backslash K$. Since the fundamental solution $\Phi$ is real analytic in a neighborhood of $\tilde{K} \times K$, there exist constants $a$ and $c$, depending on $\Phi$ and $\tilde{K}$, such that

$$
\sup _{(x, y) \in \tilde{K} \times K}\left\|D_{y}^{\alpha} \Phi(x, y)\right\| \leq c \cdot a^{|\alpha|}|\alpha| \text { ! for all } \alpha \in \mathbb{N}_{0}^{n} .
$$

Using (2), for $\alpha \in \mathbb{N}_{0}^{n}$ we get

$$
\begin{aligned}
\sup _{x \in \tilde{K}}\left|\int_{K} D_{y}^{\alpha} \Phi(x, y) c_{\alpha}(y) d m(y)\right| & \leq c \cdot a^{|\alpha|}|\alpha| ! \int_{K}\left|c_{\alpha}(y)\right| d m(y) \\
& \leq c \cdot a^{|\alpha|}|\alpha| !\left\|c_{\alpha}\right\|_{L^{p}(K, m)} m(K)^{\frac{1}{q}}
\end{aligned}
$$

with $p^{-1}+q^{-1}=1$. Therefore

$$
\begin{aligned}
& \sum_{\alpha \in \mathbb{N}_{0}^{n}} \sup _{x \in \tilde{K}}\left|\int_{K} D_{y}^{\alpha} \Phi(x, y) c_{\alpha}(y) d m\right| \leq c \cdot m(K)^{1 / q} \sum_{\alpha \in \mathbb{N}_{0}^{n}} a^{|\alpha|}|\alpha| !\left\|c_{\alpha}\right\|_{L^{p}(K, m)} \\
& \quad=c \cdot m(K)^{1 / q} \sum_{j=0}^{\infty} a^{j} \sup _{|\alpha|=j}\left\|\alpha ! c_{\alpha}\right\|_{L^{p}(K, m)}\left(\sum_{|\alpha|=j} \frac{|\alpha| !}{\alpha !}\right) \\
& \quad=c \cdot m(K)^{1 / q} \sum_{j=0}^{\infty}\left(a \cdot n \sup _{|\alpha|=j}\left\|\alpha ! c_{\alpha}\right\|_{L^{p}(K, m)}^{1 /|\alpha|}\right)^{j}
\end{aligned}
$$

where we used that $\sum_{|\alpha|=j} \frac{|\alpha| !}{\alpha !}=n^{j}, \quad n=\operatorname{dim} \mathbb{R}^{n}$.

Now, since $\sup _{|\alpha|=j}\left\|\alpha ! c_{\alpha}\right\|_{L^{p}(K, m)}^{1 / \alpha \mid} \rightarrow 0$ when $j \rightarrow \infty$, the last sum can be majorized by a geometric sum. Hence

$$
\sum_{\alpha \in \mathbb{N}_{0}^{n}} \sup _{x \in \tilde{K}}\left|\int_{K} D_{y}^{\alpha} \Phi(x, y) c_{\alpha}(y) d m\right| \leq c(K, \tilde{K})<\infty .
$$

1.5. Let us distinguish the principal difficulty in the proof of Theorem 1.1.

Lemma 1.2. Let $K$ be a locally connected compact subset of $X, m$ be a massive measure on $K$ and $1<p<\infty$. Then for every solution $f \in S_{P}(X \backslash K)$ there are a solution $f_{e} \in S_{P}(X)$ and a sequence $\left\{c_{\alpha}\right\}_{\alpha \in \mathbb{N}_{0}^{n}} \subset\left[L^{p}(K, m)\right]^{k}$ such that

$$
f(x)=f_{e}(x)+\sum_{\alpha \in \mathbb{N}_{0}^{n}} \int_{K} D_{y}^{\alpha} \Phi(x, y) c_{\alpha}(y) d m(y)
$$

holds for all $x \in X \backslash K$. Furthermore, $\left\|\alpha ! c_{\alpha}\right\|_{L^{p}(K, m)}^{1 /|\alpha|} \rightarrow 0$ when $|\alpha| \rightarrow \infty$. 
As Baernstein showed in [2], even for $P=\partial / \partial \bar{z}$ Lemma 1.2 is false for arbitrary compact $K$.

We now turn to the

Proof (of Theorem 1.1). Let $U \subset X$ be a relatively compact open set such that $U \cap \sigma=\stackrel{\circ}{K}$ and the set $K^{\prime}=\partial U \cup \stackrel{\circ}{K}$ is locally connected. Fix some massive measure $m^{\prime}$ on $K^{\prime}$ whose restriction to $K$ is $m$. The existence of such a measure follows from Example 1.1. Given a solution $f \in S_{P}(X \backslash \sigma)$, we consider the function $f^{\prime}$ which equals $f$ in $U \backslash \sigma$ and is 0 in $X \backslash \bar{U}$. Then $f^{\prime}$ is a solution of the system $P f^{\prime}=0$ with singularities on $K^{\prime}$. Hence by Lemma 1.2 there exist a solution $f_{e}^{\prime} \in S_{P}(X)$ and a sequence $\left\{c_{\alpha}^{\prime}\right\}_{\alpha \in \mathbb{N}_{0}^{n}} \subset\left[L^{p}\left(K^{\prime}, m^{\prime}\right)\right]^{k}$, satisfying $\left\|\alpha ! c_{\alpha}^{\prime}\right\|_{L^{p}\left(K^{\prime}, m^{\prime}\right)}^{1 / \alpha \mid} \rightarrow 0$ when $|\alpha| \rightarrow \infty$, such that

$$
f^{\prime}(x)=f_{e}^{\prime}(x)+\sum_{\alpha \in \mathbb{N}_{0}^{n}} \int_{K^{\prime}} D_{x}^{\alpha} \Phi(x, y) c_{\alpha}^{\prime}(y) d m^{\prime}(y) \quad\left(x \in X \backslash K^{\prime}\right) .
$$

Set $c_{\alpha}:=\left.c_{\alpha}^{\prime}\right|_{K}, \alpha \in \mathbb{N}_{0}^{n}$. Since $\left\|\alpha ! c_{\alpha}\right\|_{L^{p}(K, m)}^{1 /|\alpha|} \rightarrow 0$ when $|\alpha| \rightarrow \infty$, the function $f_{e}$ defined by

$$
f_{e}(x)=f(x)-\sum_{\alpha \in \mathbb{N}_{0}^{n}} \int_{K} D_{x}^{\alpha} \Phi(x, y) c_{\alpha}(y) d m(y) \quad(x \in X \backslash \sigma)
$$

belongs to $S_{P}(X \backslash \sigma)$ because of Lemma 1.1. Moreover, this function satisfies the equation $P f_{e}=0$ also in a neighborhood of each interior point of $K$, since we have

$$
f_{e}(x)=f_{e}^{\prime}(x)+\sum_{\alpha \in \mathbb{N}_{0}^{n}} \int_{K^{\prime} \backslash K} D_{x}^{\alpha} \Phi(x, y) c_{\alpha}^{\prime}(y) d m^{\prime}(y) \text { for } x \in U \backslash \sigma .
$$

Thus $f_{e} \in S_{P}((X \backslash \sigma) \cup \stackrel{\circ}{K})$, as was to be proved.

The proof of Lemma 1.2 needs some preparation which we give in the following section by studying more thoroughly the topology on $S_{P^{\prime}}(K)$. For the sake of simplicity, we restrict the following considerations to the case $k=1$.

\section{Equivalent topologies in $S_{P^{\prime}}(K)$}

2.1. Let $K$ be any compact set in $X$. In this section, we study various topologies on $S_{P^{\prime}}(K)$, where $P^{\prime}$ is the transposed operator to $P$. Define the space $S_{P^{\prime}}(K)$ as follows. The function $g$ belongs to $S_{P^{\prime}}(K)$ if there exists an open set $U \supset K$ such that $g$ is a solution of the equation $P^{\prime} g=0$ in $U$. If two such functions agree on some neighborhood of $K$, we identify them as elements in $S_{P^{\prime}}(K)$.

For each $U$ as above, let $S_{P^{\prime}}(U)$ denote the space of solutions of the equation $P^{\prime} g=0$ in $U$ with the topology of uniform convergence on compact subsets, i.e., the topology induced from $C(U)$. There is a natural map from $S_{P^{\prime}}(U)$ into $S_{P^{\prime}}(K)$, and we endow $S_{P^{\prime}}(K)$ with the finest locally convex topology for which all these maps are continuous. We denote this topology by $\tau$. Alternatively, the space $\left(S_{P^{\prime}}(K), \tau\right)$ can be described as the inductive limit of the spaces $S_{P^{\prime}}\left(U_{\nu}\right)$, where $\left\{U_{\nu}\right\}$ is any decreasing sequence of open sets containing $K$ such that each neighborhood of $K$ contains some $U_{\nu}$, and such that each component of each $U_{\nu}$ meets $U_{\nu+1}$. 
Remark 2.1. The space $\left(S_{P^{\prime}}(K), \tau\right)$ is separated, a subset of this space is bounded iff it is contained and bounded in some $S_{P^{\prime}}\left(U_{\nu}\right)$, and each closed bounded subset is compact. Proofs could be given by the same methods as in Koethe [9], p.379.

2.2. We will embed $S_{P^{\prime}}(K)$ algebraically in a space $L^{(q)}$ whose topological dual consists of sequences of functions from $L^{p}(K, m)$. Lemma 1.2 follows from the HahnBanach Theorem once we show that the topology of $L^{(q)}$ restricted to $S_{P^{\prime}}(K)$ is finer than the topology $\tau$. To do this, we have first to study some Banach spaces.

Definition 2.1. Given positive numbers $q$ and $r$, the space $l^{q}(r)$ is defined to consist of all sequences $\left\{\eta_{\alpha}\right\}_{\alpha \in \mathbb{N}_{0}^{n}} \subseteq \mathbb{C}$ with $\left(\sum_{\alpha \in \mathbb{N}_{0}^{k}}\left|\eta_{\alpha}\right|^{q} r^{|\alpha| q}\right)^{1 / q}<\infty$.

If $K$ is an arbitrary compact subset of $X$ and $m$ is an arbitrary measure on $K$, then we denote by $l^{q}(r)^{K}$ the space of all functions $\eta(\cdot)=\left\{\eta_{\alpha}(\cdot)\right\}_{\alpha \in \mathbb{N}_{0}^{n}}$ on $K$ with values in $l^{q}(r)$ such that $\eta_{\alpha}(\cdot) \in L^{q}(K, m)$ for every $\alpha \in \mathbb{N}_{0}^{n}$ and

$$
\left(\sum_{\alpha \in \mathbb{N}_{0}^{n}}\left\|\eta_{\alpha}\right\|_{L^{q}(K, m)}^{q} r^{q|\alpha|}\right)^{1 / q}<\infty .
$$

Lemma 2.1. For $q \in[1, \infty]$, the functional

$$
\left\|\left\{\eta_{\alpha}\right\}\right\|_{l^{q}(r)^{K}}=\left(\sum_{\alpha \in \mathbb{N}_{0}^{n}}\left\|\eta_{\alpha}\right\|_{L^{q}(K, m)}^{q} r^{q|\alpha|}\right)^{1 / q}
$$

defines a norm on $l^{p}(r)^{K}$.

Proof. The proof is an easy exercise from functional analysis.

Equipped with the norm (1), the space $l^{q}(r)^{K}$ is a Banach space, provided $q \in$ $[1, \infty]$. Instead of proving this directly, we proceed by the following

Lemma 2.2. Let $r>0, q \geq 1$ be arbitrary real numbers, and let $p \in] 1, \infty]$ be the conjugate exponent to $q$. We have an isometrical isomorphism

$$
\left(l^{q}(r)^{K}\right)^{\prime} \cong l^{p}\left(\frac{1}{r}\right)^{K} .
$$

Proof. Assume that $q>1$. Fix some $\theta=\left\{\theta_{\alpha}\right\}_{\alpha \in \mathbb{N}_{0}^{n}} \in l^{p}\left(\frac{1}{r}\right)^{K}$. Then $\theta$ defines a linear functional on $l^{q}(r)^{K}$ via $\langle\theta, \eta\rangle=\sum_{\alpha \in \mathbb{N}_{0}^{n}} \int_{K}\left\langle\theta_{\alpha}(y), \eta_{\alpha}(y)\right\rangle d m(y)$, for $\eta=$ $\left\{\eta_{\alpha}\right\} \in l^{q}(r)^{K}$. Since

$$
\begin{aligned}
|\langle\theta, \eta\rangle| & \leq \sum_{\alpha \in \mathbb{N}_{0}^{n}}\left(\left\|\theta_{\alpha}\right\|_{L^{p}(K, m)} r^{-|\alpha|}\right)\left(\left\|\eta_{\alpha}\right\|_{L^{q}(K, m)} r^{|\alpha|}\right) \\
& \leq\|\theta\|_{l^{p}\left(\frac{1}{r}\right)^{K}} \cdot\|\eta\|_{l^{q}(r)^{K}}
\end{aligned}
$$

this functional is continuous. Conversely, let $F \in\left(l^{q}(r)^{K}\right)^{\prime}$. Given a multi-index $\alpha \in$ $\mathbb{N}_{0}^{n}$, denote by $e_{\alpha}$ the element in $l^{q}(r)$ which is 1 in the $\alpha$-th entry and 0 in all other entries. On $L^{q}(K, m)$, we may define a functional by juxtaposition $g \longmapsto F\left(g e_{\alpha}\right)$ for $g \in L^{q}(K, m)$. Since $F$ is continuous, this functional is continuous, too. By duality, there is a function $\theta_{\alpha} \in L^{p}(K, m)$ such that $F\left(g e_{\alpha}\right)=\int_{K}\left\langle\theta_{\alpha}(y), g(y)\right\rangle d m(y)$ for all $g \in L^{q}(K, m)$. Since for an element $\eta=\left\{\eta_{\alpha}\right\}$ in $l^{q}(r)^{K}$ we have $\eta=\sum_{\alpha \in \mathbb{N}_{0}^{n}} \eta_{\alpha} e_{\alpha}$ and the series converges in the norm of $l^{q}(r)^{K}$, it follows that

$$
F(\eta)=\sum_{\alpha \in \mathbb{N}_{0}^{n}} F\left(\eta_{\alpha} e_{\alpha}\right)=\sum_{\alpha \in \mathbb{N}_{0}^{n}} \int_{K}\left\langle\theta_{\alpha}(y), \eta_{\alpha}(y)\right\rangle d m(y) .
$$


Put $\theta:=\left\{\theta_{\alpha}\right\}_{\alpha \in \mathbb{N}_{0}^{n}}$. To complete the proof, it remains to show that $\theta$ is in $l^{p}\left(\frac{1}{r}\right)^{K}$. To this end, we consider the sequence $\left\{\eta_{\alpha}\right\}_{\alpha \in \mathbb{N}_{0}^{n}}$ of measurable functions on $K$ given by

$$
\eta_{\alpha}:= \begin{cases}\left|\theta_{\alpha}\right|^{p-2} \bar{\theta}_{\alpha} r^{-p|\alpha|}, & \theta_{\alpha} \neq 0 \\ 0, & \theta_{\alpha}=0 .\end{cases}
$$

Since $\left|\eta_{\alpha}\right|^{q}=\left|\theta_{\alpha}\right|^{p} r^{-p q|\alpha|}$ each function $\eta_{\alpha}(\cdot)$ is in $L^{q}(K, m)$. Hence it follows

$$
\begin{aligned}
\sum_{|\alpha| \leq N}\left\|\theta_{\alpha}\right\|_{L^{p}(K, m)}^{p}\left(\frac{1}{r}\right)^{p|\alpha|} & =\left|F\left(\sum_{|\alpha| \leq N} \eta_{\alpha} e_{\alpha}\right)\right| \leq\|F\|_{\left(l^{q}(r)^{K}\right)^{\prime}}\left\|\sum_{|\alpha| \leq N} \eta_{\alpha} e_{\alpha}\right\|_{l^{q}(r)^{K}} \\
& =\|F\|_{\left(l^{q}(r)^{K}\right)^{\prime}}\left(\sum_{|\alpha| \leq N} r^{-p|\alpha|}\left\|\theta_{\alpha}\right\|_{L^{p}(K, m)}^{p}\right)^{1 / q} .
\end{aligned}
$$

Thus $\left(\sum_{|\alpha| \leq N} r^{-p|\alpha|}\left\|\theta_{\alpha}\right\|_{L^{p}(K, m)}^{p}\right)^{1 / p} \leq\|F\|_{\left(l^{q}(r)^{K}\right)^{\prime}}$ for every positive integer $N$. Together with (2) it follows that

$$
\|\theta\|_{l^{p}\left(\frac{1}{r}\right)^{K}}=\|F\|_{\left(l^{q}(r)^{K}\right)^{\prime}}
$$

as was to be proved.

For $q=1$, the proof follows the same lines with the obvious modifications.

Since the dual space to a normed space is a Banach space, Lemma 2.2 implies the following

Corollary 2.1. Let $r>0$ and $q>1$. Then $l^{q}(r)^{K}$ is a reflexive Banach space.

2.3. Note that if $r^{\prime}>r^{\prime \prime}>0$, we have a continuous embedding $l^{q}\left(r^{\prime}\right)^{K} \hookrightarrow l^{q}\left(r^{\prime \prime}\right)^{K}$. Now let $\left\{r_{\nu}\right\}_{\nu \in \mathbb{N}}$ be some decreasing sequence of positive numbers tending to zero. The space $L^{(q)}$ is defined to be the inductive limit of the spaces $l^{q}\left(r_{\nu}\right)^{K}$. The space $L^{(q)}$ is separated. Each bounded set is contained and bounded in one of the $l^{q}\left(r_{\nu}\right)^{K}$. Moreover, $L^{(q)}$ is a (DF)-space, because it is the separated inductive limit of a sequence of normed, hence (DF)-, spaces (see Théorème 9 of Grothendieck [6]).

Our aim is to show that $S_{P^{\prime}}(K)$ is topologically isomorphic to a subspace of $L^{(q)}$. Thus we proceed by constructing an embedding $S_{P^{\prime}}(K) \hookrightarrow L^{(q)}$. More precisely, for each solution $g \in S_{P^{\prime}}(K)$ we define

$$
j(g):=\left\{\left.\frac{D^{\alpha} g}{\alpha !}\right|_{K}\right\}_{\alpha \in \mathbb{N}_{0}^{n}} .
$$

Lemma 2.3. For every $g \in S_{P^{\prime}}(K)$, the sequence $j(g)$ is in $L^{(q)}$, and the mapping $j: S_{P^{\prime}}(K) \rightarrow L^{(q)}$ is continuous and injective.

Proof. Let $g \in S_{P^{\prime}}(K)$. Then there is a neighborhood $U$ of $K$ in $X$ such that $g \in S_{P^{\prime}}(U)$. Now choose a function $\varphi \in \mathcal{D}(X)$ which is equal to 1 in a neighborhood of $K$. Since $\Phi$ is a left fundamental solution of $P$, we get $g=\Phi^{\prime} P^{\prime}(\varphi g)$ in a neighborhood of $K$.

The function $P^{\prime}(\varphi g)$ is supported by the closure of the set of those points $x \in U$ such that $\operatorname{grad} \varphi(x) \neq 0$. Let us denote this closure by $\sigma$. Then $\sigma$ is a compact subset of $U \backslash K$, so there is a function $\psi \in \mathcal{D}(U \backslash K)$ which equals 1 in a neighborhood of $\sigma$.

Since $P^{\prime}(\varphi g)=\psi P^{\prime}(\varphi g)$, we have $g=\Phi^{\prime}\left(\psi P^{\prime}(\varphi g)\right)$ in a neighborhood of $K$. Hence it follows for each multi-index $\alpha$ that

$$
D^{\alpha} g(y)=\int P(x, D)\left(\psi(x) D_{y}^{\alpha} \Phi(x, y)\right) \cdot(\varphi(x) g(x)) d x \quad(y \in K) .
$$


Using estimate $(2)$ with $\tilde{K}=\operatorname{supp} \psi$, we get

$$
\begin{aligned}
\sup _{y \in K}\left|D^{\alpha} g(y)\right| & \leq c^{\prime} a^{|\alpha|+\operatorname{order} P}(|\alpha|+\operatorname{order} P) ! \sup _{x \in \operatorname{supp} \varphi}|g(x)| \\
& \leq c^{\prime \prime}\left(a^{\prime}\right)^{|\alpha|}|\alpha| ! \sup _{x \in \operatorname{supp} \varphi}|g(x)|
\end{aligned}
$$

where $a^{\prime}$ is any number larger than $a$, and the constant $c^{\prime \prime}$ does not depend on $g \in S_{P^{\prime}}(U)$ and $\alpha$. It now follows that

(4)

$$
\begin{aligned}
\sum_{\alpha \in \mathbb{N}_{0}^{n}}\left\|\frac{D^{\alpha} g}{\alpha !}\right\|_{L^{q}(K, m)}^{q} r_{\nu}^{q|\alpha|} & \leq\left(c^{\prime \prime}\right)^{q} m(K)\left(\sum_{\alpha \in \mathbb{N}_{0}^{n}}\left(\left(a^{\prime}\right)^{|\alpha|} r_{\nu}^{|\alpha|} \frac{|\alpha| !}{\alpha !}\right)^{q}\right) \sup _{x \in \operatorname{supp} \varphi}|g(x)| \\
& =\left(c^{\prime \prime}\right)^{q} m(K)\left(\sum_{j=0}^{\infty}\left(n a^{\prime} r_{\nu}\right)^{q j}\right) \sup _{x \in \operatorname{supp} \varphi}|g(x)| .
\end{aligned}
$$

Choose $\nu_{0}$ large enough, such that $\operatorname{nar}_{\nu_{0}}<1$. Then (4) shows that $j(g) \in$ $l^{q}\left(r_{\nu_{0}}\right)^{K}$ as well as the continuity of the mapping $j: S_{P^{\prime}}(U) \longrightarrow l^{q}\left(r_{\nu_{0}}\right)^{K}$.

Since a linear operator from $S_{P^{\prime}}(K)$ into a locally convex space is continuous if and only if its restriction to each $S_{P^{\prime}}(U)$ is continuous (for a proof cf. Bourbaki $[3]$ ), it follows that the mapping $j: S_{P^{\prime}}(K) \longrightarrow L^{(q)}$ is continuous.

To show that $j$ is injective let $g \in S_{P^{\prime}}(K)$ be such that $j(g)=0$. This means that $\left.D^{\alpha} g\right|_{K} \equiv 0$ in $K$ for all $\alpha \in \mathbb{N}_{0}^{n}$, and hence, since $g$ is real analytic, it follows $g \equiv 0$ in a neighborhood of $K$.

\subsection{Now put}

$$
S_{P^{\prime}}^{(q)}:=j\left(S_{P^{\prime}}(K)\right) \subseteq L^{(q)} .
$$

We endow this space with the topology induced by $L^{(q)}$. We want to show

Lemma 2.4. Let $K$ be a locally connected compact subset of $X$, and $q>1$. Then $S_{P^{\prime}}^{(q)}$ is a closed subspace of $L^{(q)}$.

For the proof of Lemma 2.4 we shall use the following result:

Lemma 2.5. Assume that $\left\{L_{\nu}\right\}$ is a sequence of reflexive Banach spaces, such that $L_{\nu}$ is continuously embedded in $L_{\nu+1}$ for all $\nu$, and $L$ is the inductive limit of the sequence. Then a vector subspace $\Sigma$ of $L$ is closed if and only if for all $\nu$ the intersection $\Sigma \cap L_{\nu}$ is closed in $L_{\nu}$.

Proof. See Makarov [11].

Proof (of Lemma 2.4). Using Lemma 2.5 it is sufficient to show that for each $\nu$ the subspace $S_{P^{\prime}}^{(q)} \cap\left(l^{q}\left(r_{\nu}\right)^{K}\right)$ is closed in $l^{q}\left(r_{\nu}\right)^{K}$.

Assume that for a solution $g \in S_{P^{\prime}}(K)$ the image $j(g)$ is in $l^{q}\left(r_{\nu}\right)^{K}$. Then for all points $y \in K$, except perhaps for a set of zero measure $m$, we have

$$
\left(\sum_{\alpha \in \mathbb{N}_{0}^{n}}\left|\frac{D^{\alpha} g(y)}{\alpha !}\right|{ }^{q} r_{\nu}^{q|\alpha|}\right)^{1 / q}<\infty .
$$

Since the measure $m$ is supposed to be massive, this inequality holds for a set $\sigma_{g}$ of points $y \in K$ which is dense in $K$. So

$$
\limsup _{|\alpha| \rightarrow \infty}\left|\frac{D^{\alpha} g(y)}{\alpha !}\right|^{1 /|\alpha|} \leq \frac{1}{r_{\nu}} \text { for all } y \in \sigma_{g} .
$$


We shall construct a complex neighborhood $U_{\nu}$ of $K$ into which all the elements of $j^{-1}\left(l^{q}\left(r_{\nu}\right)^{K}\right)$ have (single valued) holomorphic extensions. This is the only place where we use the local connectedness of $K$.

For each $y \in K$ choose a neighborhood $O_{y}$ in $\mathbb{C}^{n}$ such that $O_{y} \subset \Delta\left(y, r_{\nu}\right)$ and such that $K \cap O_{y}$ is connected. This is possible, since $K$ is assumed to be locally connected. Here $\Delta(y, r)=\left\{z \in \mathbb{C}^{n}:\left|z_{i}-y_{i}\right|<r(i=1, \ldots, n)\right\}$ is the polydisk in $\mathbb{C}^{n}$ with center $y$ and radius $r$. Choose $r_{y}$ such that $\Delta\left(y, 2 r_{y}\right) \subset O_{y}$. Define $U_{\nu}=\bigcup_{y \in K} \Delta\left(y, r_{y}\right)$. Then $U_{\nu}$ is a neighborhood of $K$ in $\mathbb{C}^{n}$.

Let $g \in j^{-1}\left(l^{q}\left(r_{\nu}\right)^{K}\right)$ and $z \in U_{\nu}$. Define $\tilde{g}(z)=\sum_{\alpha} \frac{D^{\alpha} g(y)}{\alpha !}(z-y)^{\alpha}$ where $y$ is any point of $\sigma_{g}$ such that $z \in \Delta\left(y, r_{y}\right)$. The series converges, since $\left|z_{i}-y_{i}\right|<\frac{1}{2 r_{\nu}}$ for all $i=1, \ldots, n$. We have to show that $\tilde{g}(z)$ does not depend on $y$.

Suppose that $z \in \Delta\left(y^{\prime}, r_{y^{\prime}}\right) \cap \Delta\left(y^{\prime \prime}, r_{y^{\prime \prime}}\right)$, where $y^{\prime}, y^{\prime \prime} \in \sigma_{g}$. Let $r_{y^{\prime \prime}} \leq r_{y^{\prime}}$. Then $\left|y_{i}^{\prime \prime}-y_{i}^{\prime}\right|<r_{y^{\prime}}+r_{y^{\prime}} \leq 2 r_{y^{\prime}}$ for all $i=1, \ldots, n$; hence $y^{\prime \prime} \in \Delta\left(y^{\prime}, 2 r_{y^{\prime}}\right) \subset O_{y^{\prime}}$. We conclude that both $y^{\prime}$ and $y^{\prime \prime}$ belong to the connected set $K \cap O_{y^{\prime}}$. Let $U$ be an open set in $\mathbb{C}^{n}$ containing $K$, into which $g$ has a (single valued) holomorphic extension. Then $K \cap O_{y^{\prime}} \subset U \cap \Delta\left(y^{\prime}, r_{\nu}\right)$, and we denote by $O$ the component of the set on the right which contains $y^{\prime}$. Obviously, $y^{\prime \prime}$ is in $O$, too. The equation $g(z)=\sum_{\alpha} \frac{D^{\alpha} g\left(y^{\prime}\right)}{\alpha !}\left(z-y^{\prime}\right)^{\alpha}$ is valid for all $z \in O$. Hence the series

$$
\tilde{g}(z)=\sum_{\alpha} \frac{D^{\alpha} g\left(y^{\prime \prime}\right)}{\alpha !}\left(z-y^{\prime \prime}\right)^{\alpha} \quad \text { about } y^{\prime \prime}
$$

is a rearrangement of the series

$$
g(z)=\sum_{\alpha} \frac{D^{\alpha} g\left(y^{\prime}\right)}{\alpha !}(z-y)^{\alpha} \quad \text { about } y^{\prime}
$$

and uniqueness of $\tilde{g}(z)$ follows.

It is obvious that $\tilde{g}$ is holomorphic in $U_{\nu}$. Moreover, it is easily verified that $\tilde{g}$ and $g$ agree on $U_{\nu} \cap U$. We may assume that the coefficients of the differential operator $P$ have holomorphic extensions to $U_{\nu}$. Then $P^{\prime} \tilde{g} \equiv 0$ in $U_{\nu}$, since the function $P^{\prime} \tilde{g}$ is holomorphic in $U_{\nu}$ and vanishes on an open subset of each component of $U_{\nu}$.

Thus every solution $g \in j^{-1}\left(l^{q}\left(r_{\nu}\right)^{K}\right)$ has a (single valued) extension to the complex neighborhood $U_{\nu}$ of $K$. Now, let $\left\{\eta^{(j)}\right\}$ be a sequence in $S_{P^{\prime}}^{(q)} \cap l^{q}\left(r_{\nu}\right)^{K}$ which converges to an element $\eta=\left\{\eta_{\alpha}\right\}$ in $l^{q}\left(r_{\nu}\right)^{K}$. We would like to prove that $\eta$ is in $S_{P^{\prime}}^{(q)} \cap l^{q}\left(r_{\nu}\right)^{K}$, too. By definition of $S_{P^{\prime}}^{(q)}$, for every $j=1,2, \ldots$ there is a $g_{j} \in S_{P^{\prime}}(K)$ such that $\eta_{\alpha}^{(j)}=\left.\frac{D^{\alpha} g_{j}}{\alpha !}\right|_{K} \quad\left(\alpha \in \mathbb{N}_{0}^{n}\right)$. Moreover, as was already proved, each element $g_{j}$ is represented by a holomorphic function $g_{j}(z)$ in the complex neighborhood $U_{\nu}$ of $K$ satisfying $P^{\prime} g_{j}=0$ there.

The convergence $\eta^{(j)} \rightarrow \eta$ in $l^{p}\left(r_{\nu}\right)^{K}$ means that

$$
\lim _{j \rightarrow \infty}\left(\int_{K} \sum_{\alpha \in \mathbb{N}_{0}^{n}} r_{\nu}^{q|\alpha|}\left|\frac{D^{\alpha} g_{j}(y)}{\alpha !}-\eta_{\alpha}(y)\right|^{q} d m(y)\right)^{1 / q}=0 .
$$

Hence it follows that there exists a subsequence $\left\{g_{j_{s}}\right\}$ such that for all points $y \in K$, except for a set of zero measure $m$, we have

$$
\lim _{j_{s} \rightarrow \infty}\left(\sum_{\alpha \in \mathbb{N}_{0}^{n}} r_{\nu}^{q|\alpha|}\left|\frac{D^{\alpha} g_{j_{s}}(y)}{\alpha !}-\eta_{\alpha}(y)\right|^{q}\right)^{1 / q}=0 .
$$


Since the measure $m$ is massive, equality holds for a set $\sigma$ of points $y \in K$ which is dense in $K$. We now use compactness of $K$ to conclude the following. There are a finite number of points $y^{(1)}, \ldots, y^{(n)}$ in $\sigma$ and a positive $r<r_{\nu}$ such that $K$ is contained in the union $U=\Delta\left(y^{(1)}, r\right) \cup \ldots \cup \Delta\left(y^{(n)}, r\right)$, and $\bar{U} \subset U_{\nu}$. Our purpose is to show that the sequence $\left\{g_{j_{s}}\right\}$ converges to some function $g$ in $S_{P^{\prime}}(U)$. Since the space $S_{P^{\prime}}(U)$ is complete, it suffices to prove that this sequence is a Cauchy sequence in $S_{P^{\prime}}(U)$, i.e., in each of the spaces $C(k)$, where $k$ is a compact subset of $U$. Obviously, we may restrict ourselves to compact sets $k$ lying in one of the polydisks $\Delta\left(y^{(1)}, r\right), \ldots, \Delta\left(y^{(n)}, r\right)$.

Let $k$ be a compact subset of $\Delta(y, r)$ where $\Delta(y, r)$ is one of the polydisks previously mentioned. Denote by $d$ the distance from $k$ to the $n$-skeleton of $\Delta(y, r)$, i.e., $\partial_{n} \Delta(y, r)=\left\{\zeta \in \mathbb{C}^{n}:\left|\zeta_{i}-y_{i}\right|=r(i=1, \ldots, n)\right\}$. The distance is taken in the polydisk-norm.

We may regard some branch of $\left(g_{j_{s}}(z)-g_{j_{t}}(z)\right)^{q}$ in $\Delta(y, r)$ to yield a holomorphic function there. By Cauchy's Theorem we have for all $z \in \Delta(y, r)$ :

$$
\left(g_{j_{s}}(z)-g_{j_{t}}(z)\right)^{q}=\frac{1}{(2 \pi \sqrt{-1})^{n}} \int_{\partial_{n} \Delta(y, r)} \frac{\left(g_{j_{s}}(\zeta)-g_{j_{t}}(\zeta)\right)^{q}}{\left(\zeta_{1}-z_{1}\right) \cdot \ldots \cdot\left(\zeta_{n}-z_{n}\right)} d \zeta_{1} \wedge \ldots \wedge d \zeta_{n} .
$$

The Taylor-series expansion for $\left(g_{j_{s}}(\zeta)-g_{j_{t}}(\zeta)\right)$, centered at $y$, converges uniformly in the closure of $\Delta(y, r)$. So (6) implies for $z \in k$ :

$$
\begin{aligned}
& \left|g_{j_{s}}(z)-g_{j_{t}}(z)\right| \leq\left(\frac{1}{\left.(2 \pi d)^{n} \int_{\partial_{n} \Delta(y, r)}\left|g_{j_{s}}(\zeta)-g_{j_{t}}(\zeta)\right|^{q}\left|d \zeta_{1}\right| \wedge \ldots \wedge\left|d \zeta_{n}\right|\right)^{1 / q}}\right. \\
& \quad=\left(\frac{1}{(2 \pi d)^{n}} \int_{\partial_{n} \Delta(y, r)}\left|\sum_{\alpha \in \mathbb{N}_{0}^{n}} \frac{D^{\alpha}\left(g_{j_{s}}(y)-g_{j_{t}}(y)\right)}{\alpha !}(\zeta-y)^{\alpha}\right|^{q}\left|d \zeta_{1}\right| \wedge \ldots \wedge\left|d \zeta_{n}\right|\right)^{1 / q} .
\end{aligned}
$$

Using Hölder's inequality and taking into account that $r<r_{\nu}$, we get

$$
\begin{aligned}
\sup _{z \in k}\left|g_{j_{s}}(z)-g_{j_{t}}(z)\right| & \\
\leq & \left(\frac{1}{(2 \pi d)^{n}} \int_{\partial_{n} \Delta(y, r)}\left(\sum_{\alpha \in \mathbb{N}_{0}^{n}} \frac{\left|(\zeta-y)^{\alpha}\right|^{p}}{\left|r_{\nu}^{|\alpha|}\right|^{p}}\right)^{(q / p)}\left|d \zeta_{1}\right| \wedge \ldots \wedge\left|d \zeta_{n}\right|\right)^{1 / q} \\
& \cdot\left(\sum_{\alpha \in \mathbb{N}_{0}^{n}}\left|\frac{D^{\alpha}\left(g_{j_{s}}(y)-g_{j_{t}}(y)\right)}{\alpha !} r_{\nu}^{|\alpha|}\right|^{q}\right)^{1 / q} \\
= & \left(\frac{r}{d}\right)^{n / q}\left(\sum_{\alpha}\left(\frac{r}{r_{\nu}}\right)^{p|\alpha|}\right)^{1 / p}\left(\sum_{\alpha}\left|\frac{D^{\alpha} g_{j_{s}}(y)-D^{\alpha} g_{j_{t}}(y)}{\alpha !}\right|^{q} r_{\nu}^{q|\alpha|}\right)^{1 / q} \\
\leq & \left(\frac{r}{d}\right)^{n / q}\left(\frac{r_{\nu}^{p}}{r_{\nu}^{p}-r^{p}}\right)^{n / p}\left\{\left(\sum_{\alpha} r_{\nu}^{|\alpha| q \mid} \frac{D^{\alpha} g_{j_{s}}(y)}{\alpha !}-\left.\eta_{\alpha}(y)\right|^{q}\right)^{\frac{1}{q}}+\right. \\
& \left.+\left(\sum_{\alpha} r_{\nu}^{|\alpha| q \mid} \frac{D^{\alpha} g_{j_{t}}(y)}{\alpha !}-\left.\eta_{\alpha}(y)\right|^{q}\right)^{\frac{1}{q}}\right\} .
\end{aligned}
$$

By (5) it follows that $\sup _{z \in k}\left|g_{j_{s}}(z)-g_{j_{t}}(z)\right| \rightarrow 0$ when both $j_{s}$ and $j_{t}$ tend to infinity. This is just what we wanted to prove. Thus, there is a solution $g \in S_{P^{\prime}}(U)$ such that $g_{j_{s}} \rightarrow g$ in $S_{P^{\prime}}(U)$. Because of Lemma 2.3, we obtain $\eta=j(g)$. Hence $\eta \in S_{P^{\prime}}^{(q)}$, as was to be proved.

The main result of this section consists of the following. 
Theorem 2.1. Assume that $K$ is a locally connected compact subset of $X$, and $q>1$. Then the mapping $j^{-1}: S_{P^{\prime}}^{(q)} \rightarrow S_{P^{\prime}}(K)$ is continuous.

Proof. The assertion follows from Lemma 2.4 and a version of the Open Mapping Theorem, but we prefer the direct proof. As was already mentioned, the mapping $j^{-1}: S_{P^{\prime}}^{(q)} \rightarrow S_{P^{\prime}}(K)$ is continuous, iff each restriction $j^{-1}: S_{P^{\prime}}^{(q)} \cap l^{q}\left(r_{\nu}\right)^{K}$ is continuous (see Bourbaki [3]). Let $\left\{g_{j}\right\}$ be a sequence of $S_{P^{\prime}}(K)$ such that the sequence $\left\{\frac{D^{\alpha} g_{j}}{\alpha !}\right\}_{\alpha \in \mathbb{N}_{0}^{n}}$ converges to zero in $l^{q}\left(r_{\nu}\right)^{K}$. By the same way as we proceeded in the proof of Lemma 2.4, we find a complex neighborhood $U_{\nu}$ of $K$ such that every element $g_{j}$ is represented by a holomorphic function $g_{j}(z)$ in $U_{\nu}$ satisfying $P^{\prime} g_{j}=0$ there.

Choose a positive $r<r_{\nu}$ such that the set $U=\bigcup_{y \in K} \Delta(y, r)$ is contained in $U_{\nu}$ together with its closure. Then we claim that $\left\{g_{j}\right\}$ tends to zero uniformly on compact subsets of $U$. In fact, otherwise there would exist a compact set $k \subset U$, an $\varepsilon>0$ and a subsequence $\left\{g_{j_{s}}\right\}$ such that $\sup _{z \in k}\left|g_{j_{s}}(z)\right| \geq \varepsilon$ for all $j_{s}$. But then it follows just in the same way as in the proof of Lemma 2.4 that some subsequence of $\left\{g_{j_{s}}\right\}$ should tend to zero uniformly on compact subsets of $U$. This contradiction implies our statement. Hence $g_{j} \rightarrow 0$ in $S_{P^{\prime}}(K)$, as was to be proved.

Combining Theorem 2.1 and Lemma 2.3, we obtain the

Corollary 2.2. Under the conditions of Theorem 2.1, the mapping $j: S_{P^{\prime}}(K) \rightarrow$ $S_{P^{\prime}}^{(q)}$ is a topological isomorphism of the space $\left(S_{P^{\prime}}(K), \tau\right)$ onto the space $S_{P^{\prime}}^{(q)}$ equipped with the topology induced by $L^{(q)}$.

\section{Proof of the main lemma And Remarks}

3.1. In order to prove Lemma 1.2, we shall use the fact that each solution $f \in$ $S_{P}(X \backslash K)$ may be written as the sum of a solution in $S_{P}(X)$ and a solution in $S_{P}(X \backslash K)$ which is regular at infinity. The latter notion can be introduced as follows:

Denote by $\hat{X}$ the one point compactification of $X$, i.e., the union of $X$ and the symbolic point $\infty$. The topology in $\hat{X}$ is defined by the following system of neighborhoods: If $x \in X$, then we take the usual neighborhood basis, and if $x=\infty$, then we take the family of complements of all compact subsets in $X$. Let $U$ be a neighbourhood of $\infty$. A function $f \in S_{P}(U)$ which has the representation (in a neighborhood of $\infty$, possibly smaller than $U) f=\Phi(F)$, for some distribution $F$ with compact support, in $K$, is called regular at infinity. Here $\Phi(F)$ is the value of the pseudo-differential operator $\Phi$ on $F$. For smooth functions $F$ with compact support $\Phi(F)$ is defined by $\Phi(F)=\int_{\mathbb{R}^{n}} \Phi(\cdot, y) F(y) d y$. For distributions $F$ with compact support, $\Phi(F)$ is defined by duality.

Of course, this notion depends on our particular choice of the fundamental solution $\Phi$, while the space of solutions regular at infinity does not depend on $\Phi$ on the whole.

Let us denote by $S_{P}^{(r)}(X \backslash K)$ the subspace of $S_{P}(X \backslash K)$ consisting of the solutions regular at infinity.

Lemma 3.1. For each compact set $K \subset X$, it follows that

$$
S_{P}(X \backslash K)=S_{P}(X) \oplus S_{P}^{(r)}(X \backslash K) .
$$

The sum on the right is topological. 
Proof. Let $G_{P}$ be a Green operator for $P$, i.e., a bidifferential operator of order $\operatorname{ord}(P)-1$ on $X$ with the property that $d G_{P}(g, f)=\left(\langle g, P f\rangle_{x}-\left\langle P^{\prime} g, f\right\rangle_{x}\right) d x$ for all $g$ and $f$, which are smooth enough in $X$. Here $d x=d x_{1} \wedge \ldots \wedge d x_{n}$. Given a solution $f \in S_{P}(X \backslash K)$, we define the functions $f_{e}$ and $f_{r}$ in the following way. Let $x \in X$. Choose an open set $U \subset \subset X$ with piecewise smooth boundary such that $K \subset U$ and $x \in U$. Set $f_{e}(x)=-\int_{\partial U} G_{P}(\Phi(x, \cdot), f)$. It follows from the Green formula that $f_{e}(x)$ does not depend on the particular choice of $U$. Obviously, $f_{e} \in S_{P}(X)$. Now let $x \in X \backslash K$. Let $U \subset \subset X$ be an open set with piecewise smooth boundary such that $K \subset U$ and $x \notin \bar{U}$. Set $f_{r}(x)=\int_{\partial U} G_{P}(\Phi(x, \cdot), f)$. Again, $f_{r}$ does not depend on the choice of $U$. It is clear that $f_{r} \in S_{P}^{(r)}(X \backslash K)$. By the Green formula we get $f=f_{e}+f_{r}$. The rest of the proof is obvious.

Thus, every solution $f \in S_{P}(X \backslash K)$ may be written in the form $f=f_{e}+f_{r}$, with $f_{e} \in S_{P}(X)$ and $f_{r} \in S_{P}^{(r)}(X \backslash K)$, and this representation is unique.

3.2. Given a solution $f \in S_{P}(X \backslash K)$, we define a linear functional $F_{f}$ on $S_{P^{\prime}}(K)$ as follows. Let $g \in S_{P^{\prime}}(K)$. This means that there is a neighborhood $U$ of $K$ such that $g \in S_{P^{\prime}}(U)$. Choose a new neighborhood $U_{g}$ of $K$ such that $U_{g} \subset \subset U$ and the boundary of $U_{g}$ is piecewise smooth. Put

$$
\left\langle F_{f}, g\right\rangle=\int_{\partial U_{g}} G_{P}(g, f) \quad\left(g \in S_{P^{\prime}}(K)\right) .
$$

It follows from the Green formula, that the value $\left\langle F_{f}, g\right\rangle$ does not depend on the particular choice of $U_{g}$. Moreover, $F_{f}$ is a continuous linear functional on $S_{P^{\prime}}(K)$.

Lemma 3.2. If $f \in S_{P}(X \backslash K)$, then

$$
\left\langle F_{f}, \Phi(x, \cdot)\right\rangle=f_{r}(x) \text { for } x \in X \backslash K .
$$

Proof. In fact, if $x \in X \backslash K$, then $\Phi(x, \cdot)$ satisfies $P^{\prime} \Phi(x, \cdot)=0$ in the neighborhood $X \backslash\{x\}$ of $K$. So the left-hand side of (2) is well-defined. To finish the proof, it only remains to look at the proof of Lemma 3.1.

3.3. We proceed now by applying Theorem 2.1. Therefore, we are interested in a representation of functionals $F \in\left(L^{(q)}\right)^{\prime}$, where $1<q<\infty$.

Lemma 3.3. Let $1<q<\infty$ and $p$ be the conjugate exponent to $q$. To each continuous linear functional $F$ on $\left(L^{(q)}\right)$ there is a sequence $f=\left\{f_{\alpha}\right\}_{\alpha \in \mathbb{N}_{0}^{n}}$ in $L^{p}(K, m)$ such that $\left\|f_{\alpha}\right\|_{L^{p}(K, m)}^{1 / \alpha \mid} \rightarrow 0$ when $|\alpha| \rightarrow \infty$, such that

$$
\langle F, \eta\rangle=\sum_{\alpha \in \mathbb{N}_{0}^{n}} \int_{K}\left\langle f_{\alpha}(y), \eta_{\alpha}(y)\right\rangle d m(y) \text { for all } \eta=\left\{\eta_{\alpha}\right\} \in L^{(q)} .
$$

Proof. Let $\eta \in l^{q}\left(r_{\nu}\right)^{K}$. Then $\eta=\sum_{\alpha \in \mathbb{N}_{0}^{n}} \eta_{\alpha} e_{\alpha}$, and the series converges with respect to the norm of $l^{q}\left(r_{\nu}\right)^{K}$. Since $F$ is a continuous functional on $L^{(q)}$, its restriction to each of the $l^{q}\left(r_{\nu}\right)^{K}$ is continuous, too. Therefore, we have $\langle F, \eta\rangle=$ $\sum_{\alpha}\left\langle F, \eta_{\alpha} e_{\alpha}\right\rangle$ for all $\eta=\left\{\eta_{\alpha}\right\} \in L^{(q)}$. For a fixed multi-index $\alpha$, we consider the linear functional on $L^{q}(K, m)$ defined by $g \longmapsto\left\langle F, g e_{\alpha}\right\rangle \quad\left(g \in L^{q}(K, m)\right)$. This functional is obviously continuous, so by duality there is a function $f_{\alpha} \in L^{p}(K, m)$ such that $\left\langle F, g e_{\alpha}\right\rangle=\int_{K}\left\langle f_{\alpha}(y), g(y)\right\rangle d m(y)$ for all $g \in L^{q}(k, m)$. Hence $\langle F, \eta\rangle=$ $\sum_{\alpha} \int_{K}\left\langle f_{\alpha}(y), \eta_{\alpha}(y)\right\rangle d m(y)$ for all $\eta \in L^{(q)}$. The expression on the right hand side of this equality is a continuous linear functional on $L^{(q)}$, and thus on each of the 
spaces $l^{q}\left(r_{\nu}\right)^{K}$. Hence it follows by Lemma 2.2 that $\left\{f_{\alpha}\right\} \in l^{p}\left(\frac{1}{r_{\nu}}\right)^{K}$ for every $\nu$. Then $\sum_{\alpha}\left(\left\|f_{\alpha}\right\|_{L^{p}(K, m)}^{1 /|\alpha|} \frac{1}{r_{\nu}}\right)^{p|\alpha|}<\infty$, showing that $\lim \sup _{|\alpha| \rightarrow \infty}\left\|f_{\alpha}\right\|_{L^{p}(K, m)}^{1 / \alpha \mid} \leq r_{\nu}$ for all $\nu$. Since $r_{\nu} \rightarrow \infty$, the assertion follows.

\subsection{We now turn to the}

Proof (of Lemma 1.2). Assume that $f \in S_{P}(X \backslash K)$. We consider the continuous linear functional $F_{f}$ on $S_{P^{\prime}}(K)$ given by formula (1). The composition $F=F_{f} \circ j^{-1}$ defines a linear functional on the space $S_{P^{\prime}}^{(q)}$, as follows from Lemma 2.3. Because of Theorem 2.1, the functional $F$ is continuous. By the Hahn-Banach Theorem, $F$ can be continuously extended to the whole space $L^{(q)}$. According to Lemma 3.3, there exists a sequence $\left\{f_{\alpha}\right\}_{\alpha \in \mathbb{N}_{0}^{n}}$ in $L^{p}(K, m)$, satisfying $\left\|f_{\alpha}\right\|_{L^{p}(K, m)}^{1 /|\alpha|} \rightarrow 0$ when $|\alpha| \rightarrow \infty$, such that

$$
\langle F, j(g)\rangle=\sum_{\alpha} \int_{K}\left\langle f_{\alpha}(y), \frac{D^{\alpha} g(y)}{\alpha !}\right\rangle d m(y) \text { for all } g \in S_{P^{\prime}}(K) .
$$

Now putting $g=\Phi(x, \cdot)$, where $x$ is a fixed point of $X \backslash K$, and using Lemma 3.2 we derive the assertion of Lemma 1.2 with $c_{\alpha}=f_{\alpha} / \alpha !\left(\alpha \in \mathbb{N}_{0}^{n}\right)$, since

$$
\langle F, j(\Phi(x, \cdot))\rangle=\left\langle F_{f}, \Phi(x, \cdot)\right\rangle=f_{r}(x)=f(x)-f_{e}(x)
$$

3.5. When $K$ is a single point, the representation asserted by Lemma 1.2 is just the Laurent expansion of $f$.

Corollary 3.1. Let $y_{0}$ be a fixed point of $X$. Then for every solution $f \in$ $S_{P}\left(X \backslash\left\{y_{0}\right\}\right)$ there exist a solution $f_{e} \in S_{P}(X)$ and a sequence $\left\{c_{\alpha}\right\}_{\alpha \in \mathbb{N}_{0}^{n}} \subset \mathbb{C}^{k}$, satisfying $\left|\alpha ! c_{\alpha}\right|^{1 /|\alpha|} \rightarrow 0$ when $|\alpha| \rightarrow \infty$, such that

$$
f(x)=f_{e}(x)+\sum_{\alpha} D_{y}^{\alpha} \Phi\left(x, y_{0}\right) c_{\alpha}\left(x \in X \backslash\left\{y_{0}\right\}\right)
$$

Proof. The assertion follows by using $m\left(y_{0}\right)=1$ as a massive measure on $K=$ $\left\{y_{0}\right\}$.

The coefficients $\left\{c_{\alpha}\right\}$ will not be uniquely determined by $f$, since

$$
P^{\prime}\left(y_{0}, D_{y}\right) \Phi\left(x, y_{0}\right)=\delta\left(x-y_{0}\right) I_{k}
$$

becomes zero off $y_{0}$.

The Laurent-series expansions for solutions of general elliptic equations were first studied by Lopatinskii [10] .

3.6. If $O \subset \subset X$ is an open set whose boundary is locally connected, then each solution $f$ of $P f=0$ in $O$ has a representation (1) for $x \in O$ with $K=\partial O$. The only thing we have to do is to construct a massive measure $m$ on $\partial O$, and to extend $f$ to a function satisfying the equation in the complement of $\partial O$. The assertion follows by Lemma 1.2. 
3.7. Theorem 1.1 implies that arbitrary singularities of solutions of elliptic equations may be locally separated into atomic (i.e. one-point) singularities.

Corollary 3.2. Assume that $K$ is a locally connected compact subset of $\sigma$, and $\left\{y_{\nu}\right\}$ is a dense sequence of points of $K$. Then every solution $f \in S_{P}(X \backslash \sigma)$ can be written in the form $f=f_{e}+\sum_{\nu} f_{\nu}$, where $f_{e} \in S_{P}((X \backslash \sigma) \cup \stackrel{\circ}{K})$ and $f_{\nu} \in S_{P}\left(X \backslash\left\{y_{\nu}\right\}\right)$, and the series converges in the topology of $S_{P}(X \backslash K)$.

Proof. We use the massive measure $m$ on $K$ constructed in Example 1.1. By Theorem 1.1

$$
f(x)=f_{e}(x)+\sum_{\alpha}\left(\sum_{\nu} D_{y}^{\alpha} \Phi\left(x, y_{\nu}\right) c_{\alpha}\left(y_{\nu}\right) \mu_{\nu}\right) \text { for } x \in X \backslash \sigma,
$$

where $f_{e} \in S_{P}((X \backslash \sigma) \cup \stackrel{\circ}{K})$ and $\lim _{|\alpha| \rightarrow \infty}\left(\sum_{\nu}\left|\alpha ! c_{\alpha}\left(y_{\nu}\right)\right|^{p} \mu_{\nu}\right)^{1 /(p|\alpha|)}=0$. The last condition allows to rearrange the summations and to derive $f=f_{e}+\sum_{\nu} f_{\nu}$ with

$$
f_{\nu}=\sum_{\alpha} D_{y}^{\alpha} \Phi\left(x, y_{\nu}\right) c_{\alpha}\left(y_{\nu}\right) \mu_{\nu}
$$

as was to be proved.

3.8. For the Laplace operator we obtain the following result (which seems to be new).

Corollary 3.3. Let $K \subset \sigma$ be a locally connected compact set, and $1<p<\infty$. Then every harmonic function $f$ in $X \backslash \sigma$ has the form

$$
f(x)=f_{e}(x)+\sum_{j=0}^{\infty} \int_{K} \frac{h_{j}(y, x-y)}{|x-y|^{n+2(j-1)}} d m(y) \quad(x \in X \backslash \sigma)
$$

where $f_{e}$ is a harmonic function in $(X \backslash \sigma) \cup \stackrel{\circ}{K}$, and $h_{j}(y, z)$ are homogeneous harmonic polynomials of degree $j$ in $z$ with coefficients in $L^{p}(K, m)$ with respect to $y$, such that $\lim _{j \rightarrow \infty}\left(\frac{1}{j !} \int_{K}\left|h_{j}\left(y, D_{z}\right) h_{j}(y, z)\right|^{p / 2} d m(y)\right)^{1 / p_{j}}=0$.

Proof. It suffices to transform formula (1) by means of the Hecke identity (cf. Stein [14]).

3.9. We finish this section by mentioning one more aspect of Theorem 1.1. It is a natural question to ask whether a given solution $f \in S_{P}\left(X \backslash\left\{y_{0}\right\}\right)$ admits a representation (3) with a finite number of summands. This is obviously the case iff $f$ has a finite order of growth near $y_{0}$, i.e., $|f(x)| \leq c\left|x-y_{0}\right|^{-\gamma}$ in some deleted neighborhood of $y_{0}$. In other words, $y_{0}$ has to be a pole of $f$. Therefore, the solutions $f \in S_{P}(X \backslash K)$ for which the expansions (1) have only a finite number of terms are analogues of solutions with poles in general. Such solutions can be characterized as follows.

Theorem 3.1. Let $K$ be an arbitrary compact set in $X, m$ be a massive measure on $K$, and $1<p<\infty$. A solution $f \in S_{P}(X \backslash K)$ has a representation (1) with a finite number of terms iff the functional $F_{f}$ given by (1) is continuous on $S_{P^{\prime}}(K)$ with respect to the topology defined by the family of seminorms $\left\|D^{\alpha} g\right\|_{L^{q}(K, m)}\left(\alpha \in \mathbb{N}_{0}^{n}\right)$.

Proof. See Tarkhanov [15]. 


\section{REFERENCES}

1. Baernstein, A.: Representations of holomorphic functions by boundary integrals. Trans. Amer. Math. Soc. 160 (1971), 27-37. MR 44:415

2. Baernstein, A.: A representation theorem for functions holomorphic off the real axis. Trans. Amer. Math. Soc. 165 (1972), 159-165. MR 45:2190

3. Bourbaki, N.: Topological vector spaces. Springer-Verlag, Berlin, Heidelberg, New York, 1987. MR 88g:46002

4. Fischer, B.; Tarkhanov, N.N.: A representation of solutions with singularities. Contemp. Math., vol. 212, Amer. Math. Soc., Providence, RI, 1998. CMP 98:05

5. Gramsch, B.: Über das Cauchy-Weil Integral für Gebiete mit beliebigem Rand. Arch. Math. (Basel) 28 (1977), 409-421. MR 58:17206

6. Grothendieck, A.: Sur les espaces (F) and (DF). Summa Brasil. Math. 3 (1954), 57-123. MR 17:765b

7. Havin, V.P.: An analogue of the Laurent series, in: Investigations in modern problems of the theory of functions of a complex variable. Fizmatgiz, Moscow 1961, 121-131 (Russian).

8. Havin, V.P.: Golubev series and the analyticity on a continuum, in: Linear and complex analysis problem book. Springer Lecture Notes 1043. Springer-Verlag, Berlin, Heidelberg, New York 1984. MR 85k:46007

9. Köthe, G.: Topologische lineare Räume I. Springer-Verlag, Berlin, Heidelberg, New York, 1960. MR 24:A411

10. Lopatinskii, Ya. B.: Behaviour of solutions of a linear elliptic system in a neighborhood of an isolated singular point. Dokl. Akad. Nauk SSSR 79 (1951) 5, 727-730 (Russian).

11. Makarov, B.M.: Inductive limits of normed spaces. Dokl. Akad. Nauk SSSR 119 (1958) 6, 1092-1094 (Russian). MR 20:5412

12. Rogers, J.T.; Zame, W.R.: Extension of analytic functions and the topology in spaces of analytic functions. Indiana Univ. Math. J. 31 (1982) 6, 809-818. MR 83k:30050

13. Simonova, S.: A representation theorem for functions harmonic off a hyperplane. Sibirsk. Mat. Zh. 34 (1993) (Russian)

14. Stein, E.M.: Singular integrals and differentiability properties of functions. Princeton University Press, Princeton 1970.

15. Tarkhanov, N.N.: The structure of solutions of elliptic systems with a compact set of singularities. Izv. VUZ. Mat. 1989 no. 12, 47-56 (Russian). MR 91e:35090

16. Tarkhanov, N.N.: Laurent series for solutions of elliptic systems. Nauka, Novosibirsk 1991 (Russian). MR 94e:35013

17. Varfolomeev, A.L.: Analytic continuation from a continuum onto its neighborhood; in: Zap. Nauchni. Sem. Leningrad. Otdel. Mat. Inst. Stekl. (LOMI) 113 (1981), 27-40 (Russian). MR 83e:30003

Max-Planck-Arbeitsgruppe, "Partielle Differentialgleichungen und Komplexe Analysis", Universität Potsdam, Am Neuen Palais 10, D - 14415 Potsdam, Germany

E-mail address: christoph@mpg-ana.uni-potsdam.de

E-mail address: tarkhan@mpg-ana.uni-potsdam.de 